\title{
Prognostic Significance of MicroRNA-21 Expression in Patients with Unresectable Metastatic Colon Cancer
}

\author{
YASUHITO ISEKI, MASATSUNE SHIBUTANI, KIYOSHI MAEDA, HISASHI NAGAHARA, \\ TATSUNARI FUKUOKA, TETSURO IKEYA, SHINJI MATSUTANI, KENJIRO KIMURA, \\ TAKAHIRO TOYOKAWA, RYOSUKE AMANO, HIROAKI TANAKA, \\ KAZUYA MUGURUMA, KOSEI HIRAKAWA and MASAICHI OHIRA \\ Department of Surgical Oncology, Osaka City University Graduate School of Medicine, Osaka, Japan
}

\begin{abstract}
Background/Aim: MicroRNA (miR)-21 is overexpressed in most solid tumors and a high expression of miR-21 in tumor tissue is associated with a poor clinical outcome. The aim of this study was to clarify the association between the miR-21 expression in the tumor and the chemotherapeutic outcomes and survival for unresectable metastatic colon cancer (CC). Materials and Methods: Formalin-fixed, paraffin-embedded (FFPE) samples of primary tumor were obtained from 32 patients who underwent primary tumor resection and received palliative chemotherapy for unresectable metastatic CC. MiR-21 was extracted from the FFPE and the expression of miR-21 was evaluated using quantitative real time-polymerase chain reaction (RT-PCR). The expression of miR-21 was calculated with 2- $\Delta \Delta C T$. Results: A high miR-21 expression was associated with reduced progression-free survival (PFS) $(p=0.0109)$ and tended to reduce overall survival $(O S)$ ( $p=0.0675)$. Conclusion: miR-21 expression might be a useful prognostic marker for chemotherapeutic outcome and survival in patients with unresectable metastatic CC.
\end{abstract}

Colorectal cancer is the third leading cause of cancer-related death in Japan (1). In particular, the clinical outcomes of unresectable metastatic colorectal cancer are very poor; the median survival time for unresectable metastatic colorectal cancer is about 30 months $(2,3)$. Surgery is recommended for colorectal cancer with distant metastasis, if both primary and metastatic tumors are resectable. However, if the

Correspondence to: Masatsune Shibutani, MD, Ph.D., Osaka City University Graduate School of Medicine, Department of Surgical Oncology, 1-4-3 Asahi-machi Abeno-ku, Osaka, 545-8585, Japan. Tel: +81 666453838, Fax: +81 666466450, e-mail: fbxbj429@ybb.ne.jp

Key Words: miR-21, prognosis, unresectable metastatic colon cancer, chemotherapeutic outcome. primary or metastatic tumors are unresectable, chemotherapy is recommended (4). Although new anticancer agents and molecular-targeted drugs improve the efficacy of chemotherapy for colorectal cancer, there are some cases in which the chemotherapeutic outcome is poor, resulting in early death $(2,3)$. Therefore, further examination to identify biomarkers associated with the aggressiveness of cancer progression or resistance to chemotherapy are required.

MicroRNAs are non-coding small RNAs (18-25 nucleotides) that combine with specific 3'-untranslated regions (3'-UTRs) on mRNA to induce mRNA degradation and inhibit protein translation (5). The expression of microRNAs is differentiated between tumor and normal tissue; it has been reported that microRNAs are associated with carcinogenesis and development of carcinomas (6). In particular, microRNA (miR)-21 is more highly expressed in tumors than in normal tissue (6-11). MiR-21 targets the mRNA of programmed cell death 4 (PDCD4), phosphatase and tensin homolog deleted from chromosome 10 (PTEN), transforming growth factor beta receptor 2 (TGFBR2), reversion-inducing cysteine-rich protein with Kazal motif $(R E C K)$, etc. and plays important roles in invasion, proliferation, dedifferentiation, metastasis and resistance to chemotherapy (7-11). Furthermore, a high expression of miR21 is associated with a poor survival (8-10).

There are no previous studies regarding the correlation between the miR-21 expression and the prognosis for unresectable metastatic colon cancer and, thus, the significance of miR-21 expression for unresectable metastatic colon cancer is still unclear. The aim of this study was to clarify the association between miR-21 expression and chemotherapeutic outcomes and survival.

\section{Materials and Methods}

Patients. A total of 32 patients who underwent surgery for primary tumors with unresectable metastatic colon cancer at the Department 
of Surgical Oncology, Osaka City University, Japan, between April 2005 and December 2013, were evaluated. The median follow-up period was 28.3 months (range=5.8-63.2). All patients underwent palliative chemotherapy with oxaliplatin (OX) or irinotecan (IRI) plus 5-fluorouracil (5-FU)/leucovorin (LV) or a prodrug of 5-FU as first-line chemotherapy. We obtained written informed consent from the patients for participation and the study protocol was approved by the ethics committee of Osaka City University. Our investigation was conducted according to the principles expressed in the Declaration of Helsinki. All patients were followed up until July 31, 2015 , or until death.

Response evaluations for chemotherapy were performed every eight weeks. A variation of approximately one week was regarded as allowable error. All patients were followed up with a physical examination, blood tests, including measurements of the levels of tumor markers, such as carcinoembryonic antigen (CEA) and carbohydrate antigen 19-9 (CA19-9), computed tomography (CT) and ultrasonography. Some patients underwent positron emission tomography or colonoscopy, where needed. The objective tumor response was assessed using the Response Evaluation Criteria in Solid Tumors (RECIST) for all patients (12).

Regarding progression-free survival (PFS), progression was noted as cancer progression and deaths of all causes. The survival time was measured from the date of the operation to the date of being lost to follow-up, date of progression or date of death due to all causes, whichever occurred first, censored at the date of last follow-up for 31 July, 2015, and progression-free patients.

Concerning overall survival (OS), deaths noted to be of all causes. The survival time was measured from the date of operation to the date of being lost to follow-up, date of death due to all causes or July 31, 2015, whichever occurred first.

Assessment of the miR-21 expression. All tissue specimens were fixed in $10 \%$ buffered formalin and embedded in paraffin. Extraction of total RNA from the formalin-fixed paraffin-embedded (FFPE) samples was performed on $15 \mu \mathrm{m}$ paraffin sections of each of the colon cancer and normal tissue samples. The slides were deparaffinized in xylene and washed in ethanol. The tumor tissues were macrodissected and total RNA was extracted from the specimens using the RecoverAll Total Nucleic Acid Isolation Kit for FFPE (Life Technology, Carlsbad, CA, USA), according to the manufacturer's instructions. Quantification of total RNA was confirmed using a NanoDrop ND-1000 spectrometer (NanoDrop, Wilmington, DE, USA) and an optical density (OD) of $260 \mathrm{~nm}$ and OD 260/280 ratio were utilized for quality control and to measure the concentration of RNA.

Complementary DNA (cDNA) was synthesized using microRNA primers with the Taqman microRNA Assay (Applied Biosystems, Foster City, CA, USA) for miR-21 and endogenous control, RNU44 and the Taqman MicroRNA Reverse Transcription Kit (Applied Biosystems) were used. Quantitative real time-polymerase chain reaction (qRT-PCR) was performed using a Taqman ${ }^{\circledR}$ microRNA Assay (Applied Biosystems) for miR-21 and RNU44 as the primers for PCR. The qRT-PCR analysis was carried out using a Step One Plus Real-Time PCR System (Applied Biosystems). The PCR mix, containing Taqman ${ }^{\circledR}$ Universal MasterMIX II (Applied Biosystems) was processed as follows: preliminary denaturation at $95^{\circ} \mathrm{C}$ for 10 min and $95^{\circ} \mathrm{C}$ for $15 \mathrm{sec}$, followed by annealing at $60^{\circ} \mathrm{C}$ for $60 \mathrm{sec}$ and elongation at $70^{\circ} \mathrm{C}$ for $30 \mathrm{sec}$ for up to 40 cycles. The cycle threshold $(\mathrm{Ct})$ for miR-21 and RNU44 from each sample was
Table I. Patients' characteristics.

\begin{tabular}{lc}
\hline Clinicopathological factors & Number \\
\hline Gender (male:female) & $19: 13$ \\
Age (Median (range), years) & $62(40-80)$ \\
Histology (well, moderately:mucinous, poorly) & $28: 4$ \\
Depth of tumor (T1-3:4) & $11: 21$ \\
Lymph node metastasis(negative:positive) & $2: 30$ \\
Lymph vessel invasion (negative:positive) & $2: 28$ \\
Blood vessel invasion (negative:positive) & $14: 16$ \\
Liver metastasis (negative:positive) & $6: 26$ \\
Lung metastasis (negative: positive) & $21: 11$ \\
Peritoneal dissemination (negative:positive) & $21: 11$ \\
Number of organs affected by metastasis (1: $\geq 2)$ & $14: 18$ \\
microRNA-21 expression (<8.1: $\geq 8.1)$ & $28: 4$ \\
Chemotherapy regimen (FOLFOX, CapeOX:FOLFIRI) & $27: 5$ \\
Molecular targeted agent (negative:positive) & $19: 13$ \\
\hline
\end{tabular}

miR-21, MicroRNA-21; FOLFOX, oxaliplatin (OX) + 5-fluorouracil (5$\mathrm{FU}$ )/leucovorin (LV); CapeOX, capecitabine+OX; FOLFIRI, irinotecan $+5-\mathrm{FU} / \mathrm{LV}$.

evaluated and the $2^{-\Delta \Delta \mathrm{Ct}}$ value, such as the relative miR-21 expression level, was calculated. The following formula was used to calculate $\Delta \Delta \mathrm{Ct}: \Delta \Delta \mathrm{Ct}=\Delta \mathrm{Ct}_{\mathrm{miR}-21}-\Delta \mathrm{Ct}_{\mathrm{RNU} 44}$. Each sample was examined in triplicate.

Statistical analysis. The differences between the groups were analyzed using the $\chi^{2}$ test, Fisher's exact test and Student's $t$-test. The duration of survival was calculated according to the KaplanMeier method. Differences in the survival curves were assessed with the log-rank test. A multivariate analysis of the clinicopathological factors associated with survival was performed using a Cox proportional hazards model. A $p$-value of $<0.05$ was considered to indicate statistical significance. The JMP 12 software program (SAS Institute, Cary, NC, USA) was used for all statistical analyses.

\section{Results}

Patients' characteristics. We set 8.1 as the cut-off value of relative expression level of miR-21 in this study and classified the patients into high $(\geq 8.1)$ and low miR-21 expression $(<8.1)$ groups. We determined this value to be a good cut-off value for the PFS. The clinicopathological characteristics of patients are shown in Table I. The study population consisted of 19 male and 13 female patients with a median age of 62 years (range=40-80). Twenty-eight patients had low-grade tumors (such as well-differentiated adenocarcinoma or moderately differentiated adenocarcinoma); the four remaining patients had high-grade tumors (such as poorly differentiated adenocarcinoma or mucinous adenocarcinoma). Twenty-six patients had liver metastasis, 11 lung metastasis, 11 peritoneal dissemination and nine distant lymph node metastasis. According to the number of sites of metastases, the single organ was observed in 14 patients and two or more organs were observed in 18 patients. 
Table II. Relationship between microRNA-21 expression and various clinicopathological features in the 32 colon cancer patients.

\begin{tabular}{lccl}
\hline & & miR-21 Level \\
\cline { 2 - 4 } & $<8.1(\mathrm{~N}=28)$ & $\geq 8.1(\mathrm{~N}=4)$ & $p$-Value \\
\hline Gender (Male:Female) & $13: 15$ & $0: 4$ & 0.0328 \\
Age (median (range), years) & $62(40-80)$ & $66(48-80)$ & 0.6552 \\
Histology (Well, moderately:poorly, signet, mucinous) & $25: 3$ & $3: 1$ & 0.4597 \\
Tumor depth (T1-3:T4) & $10: 18$ & $1: 3$ & 0.6659 \\
Lymph node metastasis (negative:positive) & $2: 26$ & $0: 4$ & 0.4572 \\
Lymphatic vessel invasion (negative:positive) & $2: 24$ & $0: 4$ & 0.4409 \\
Blood vessel invasion (negative:positive) & $12: 14$ & $2: 2$ & 0.8860 \\
Liver metastasis (negative:positive) & $5: 23$ & $1: 3$ & 0.7402 \\
Peritoneal dissemination (negative:positive) & $20: 8$ & $1: 3$ & 0.0745 \\
Lung metastasis (negative:positive) & $19: 9$ & $2: 2$ & 0.4914 \\
Chemotherapy (FOLFOX, CapeOX : FOLFIRI) & $24: 4$ & $3: 1$ & 0.6018 \\
Number of organs affected by metastasis (1: $\geq 2)$ & $13: 15$ & $1: 3$ & 0.4068 \\
Molecular-targeted agent (negative: positive) & $14: 14$ & $3: 1$ & 0.3371 \\
\hline
\end{tabular}

miR-21, MicroRNA-21; FOLFOX, oxaliplatin (OX) + 5-fluorouracil (5-FU)/leucovorin (LV); CapeOX, capecitabine+OXp; FOLFIRI, irinotecan +5 -FU/LV; EGFR, epidermal growth factor receptor; VEGF, vascular endothelial growth factor.

All patients underwent combination chemotherapy with OX or IRI plus 5-FU/LV or a prodrug of 5-FU as first-line chemotherapy. The administered chemotherapy regimens were as follows: OX plus 5-FU/LV (FOLFOX) $(n=19)$, IRI plus 5-FU/LV (FOLFIRI) $(n=5)$ and capecitabine plus OX (CapeOX) $(n=8)$. Four patients underwent chemotherapy combined with an anti-epidermal growth factor receptor (EGFR) agent and nine patients underwent chemotherapy combined with an antivascular endothelial growth factor (VFGF) agent. Four patients were in the high miR-21 expression group and 28 patients were in the low miR-21 expression group.

Correlation between the miR-21 expression and clinicopathological factors. MiR-21 expression exhibited a significant relationship with gender $(p=0.0328)$ (Table II). The remaining clinicopathological factors showed no significant relationships with miR-21 expression (Table II).

Correlation between the miR-21 expression and response to chemotherapy. A low miR-21 expression did not correlate with the objective response rate $(p=0.4914)$ or disease control rate $(p=0.6018)$ (Table III).

Survival analysis according to the miR-21 expression. There was a significant difference in the PFS between patients with a high miR-21 expression and those with a low miR-21 expression ( $p=0.0109)$ (Figure 1A). MiR-21 expression tended to correlate with overall survival (OS) $(p=0.0675)$ (Figure 1B).

The correlations between the clinicopathological factors and clinical outcomes were assessed using a COX
Table III. Correlation between miR-21expression and effect of chemotherapy.

\begin{tabular}{lccc}
\hline & \multicolumn{3}{c}{ miR-21 } \\
\cline { 2 - 4 } & High expression & Low expression & $p$-Value \\
\hline CR & 0 & 2 & \\
PR & 2 & 17 & \\
SD & 1 & 5 & \\
PD & 1 & 4 & 0.4914 \\
Objective response rate & $50.00 \%$ & $67.90 \%$ & 0.6018 \\
Disease control rate & $75.00 \%$ & $88.90 \%$ & \\
\hline
\end{tabular}

miR-21, MicroRNA-21; CR, complete response; PR, partial response; $\mathrm{SD}$, stable disease; $\mathrm{PD}$, progressive disease.

proportional hazards model. In the univariate analyses, miR21 expression showed a significant difference in association with PFS ( $p=0.0364$ ) (Table IV). In the multivariate analysis, histology, peritoneal dissemination and number of organs affected by metastasis, which are well-known prognostic factors for Stage IV colorectal cancer, were added as covariates. The multivariate analysis demonstrated that miR21 expression was an independent risk factor for PFS (hazard ratio $(\mathrm{HR})=4.927,95 \%$ confidence interval $(\mathrm{CI})=1.079$ 20.806; $p=0.0403$ ) (Table IV).

MiR-21 expression tended to be associated with OS $(p=0.0675)$ (Figure 1). The multivariate analysis demonstrated that miR-21 expression was not an independent risk factor for OS $(\mathrm{HR}=2.522,95 \% \mathrm{CI}=0.649-8.341, p=0.1681)($ Table V). 

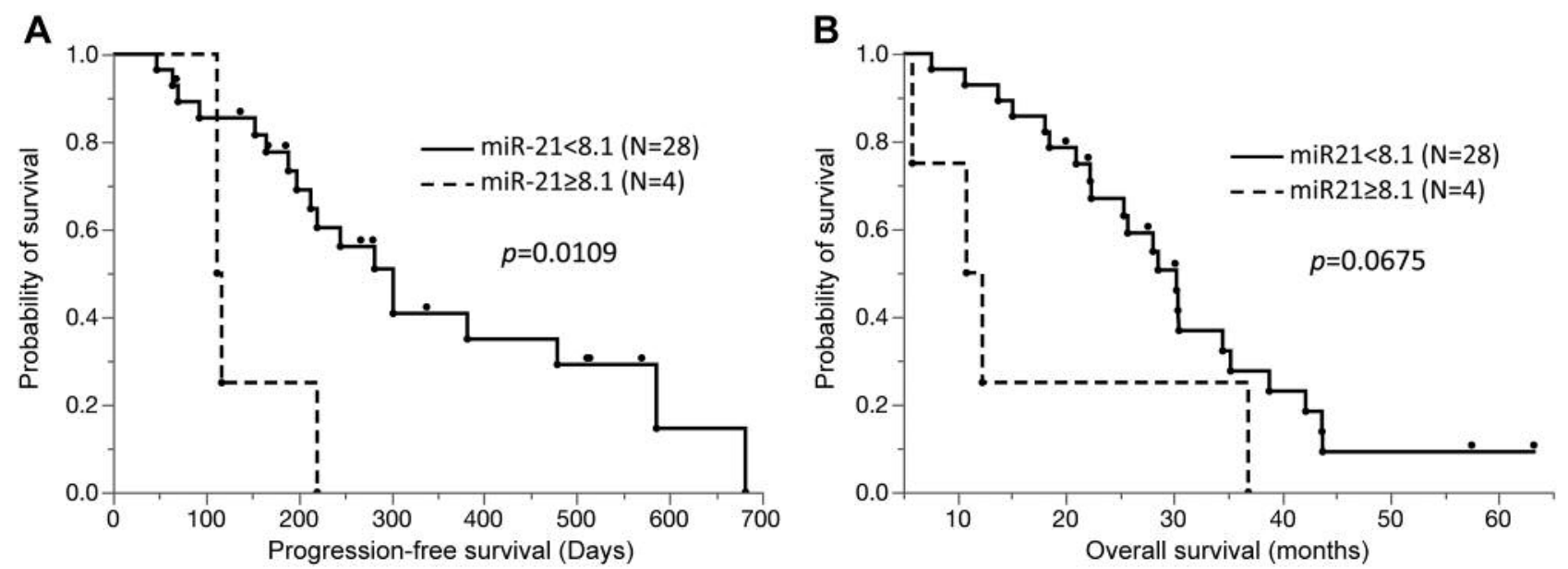

Figure 1. Survival curves for microRNA(miR)-21 expression. (A) A high miR-21 expression was associated with poorer prognosis than a low miR21 expression for progression-free survival $(p=0.0109)$. (B) A high miR-21 expression tended to be a poorer prognostic factor than a low miR-21 expression for overall survival $(p=0.0675)$.

\section{Discussion}

MicroRNA, a small non-coding RNA with 18-25 nucleotides, binds to the 3'-untranslated regions (3'-UTRs) of target mRNA to regulate gene expression by degrading mRNA and inhibiting translation (4). In particular, the expression of microRNAs in tumor tissues is different from that seen in normal tissues (5). It is reported that increased or decreased microRNAs' expression is associated with tumorigenesis and tumor progression, as various microRNAs play roles as onco-miR or tumor suppressor-miR (5). MiR-21, the expression of which is increased in tumor tissues, including colorectal cancer, is an onco-miR (5-10). MiR-21 induces tumor progression, dedifferentiation, metastasis and invasion by mediating various tumor suppressor genes (6-10).

In this study, although the miR-21 expression did not correlate with response rate of the tumor, a high miR-21 expression level correlated with a poor PFS. Moreover, according to the multivariate analysis, miR-21 expression was found to be an independent prognostic factor for PFS. MiR-21 expression may, thus, influence the time until tumor progression but not the radiological response to chemotherapy.

Various targets of miR-21, which are associated with resistance to chemotherapy, have been investigated $(6,8,23)$. Human DNA MutS homolog 2 (hMSH2) is a direct target of miR-21 (6). HMSH2 is a core mismatch repair (MMR) protein associated with MMR systems, that plays a role in recognizing DNA damage and repairing DNA (12). Because MMR impairment is inhibited as a result of the uptake of 5FU metabolites into DNA, the loss of hMSH2 induces MMR impairment, which, subsequently, reduces $\mathrm{G}_{2} / \mathrm{M}$ arrest and apoptosis associated with 5-FU chemotherapy, resulting in resistance to chemotherapy.

Phosphatase and tensin homologue deleted on chromosome $10($ PTEN), which dephosphorylates phosphatidylinositol (3,4,5)-triphosphate (PIP3) to phosphatidylinositol(4,5)diphosphate (PIP2), is associated with the PI3K/AKT pathway as a tumor suppressor $(15,16)$. The PI3K/AKT pathway is associated with the efficacy to reduce chemotherapy outcome $(16,17)$. PTEN mRNA is a direct target of miR-21. Hence, miR-21 is associated with resistance to chemotherapy and tumor proliferation by inhibiting the PTEN expression and activating the PI3K/AKT pathway (23).

When cancer cells become resistant to chemotherapy, the population of cancer stem cells in tumor tissue is increased (18). The binding of transforming growth factor (TGF) to the transforming growth factor beta receptor 2 (TGFBR2) causes phosphorylation of the transforming growth factor beta receptor 1 (TGFBR1) and phosphorylated TGFBR1 inhibits the expression of $\beta$-catenin via the SMAD pathway $(8,20)$. $\beta$ catenin plays an important role in the maintenance and proliferation of cancer stem cells via the $\mathrm{Wnt} / \beta$-catenin pathway (19). TGFBR2 mRNA is a target of miR-21 and, thus, miR-21 inhibits the expression of TGFBR2 (28). Moreover, miR-21 activates the Wnt/ $\beta$-catenin pathway, which causes an increase in the amount of cancer stem cells; that is to say, the cancer cells obtain resistance to chemotherapy by miR- 21 . Therefore, miR-21, present in cancer cells, is related to resistance to chemotherapeutic agents via various mechanisms.

MiR-21 expression correlated with a poor survival via not only resistance to chemotherapy, but also cancer progression itself. 
Table IV. Results of the uni/multivariate analysis for progression-free survival.

\begin{tabular}{|c|c|c|c|c|c|c|}
\hline & \multicolumn{3}{|c|}{ Univariate } & \multicolumn{3}{|c|}{ Multivariate } \\
\hline & HR & $95 \% \mathrm{CI}$ & $p$-Value & HR & $95 \% \mathrm{CI}$ & $p$-Value \\
\hline Age $(\geq 65$ vs. $<65)$ & 0.893 & $0.352-2.131$ & 0.8018 & & & \\
\hline Depth of tumor (T4 vs. T1-3) & 1.100 & 0.441-3.109 & 0.8445 & & & \\
\hline Histology (mucinous, poorly $v s$. well, moderately) & 1.268 & $0.288-4.011$ & 0.7206 & 0.596 & $0.089-3.016$ & 0.5481 \\
\hline Lymphatic vessel invasion (positive $v s$. negative) & 0.270 & $0.046-5.125$ & 0.3007 & & & \\
\hline Blood vessel invasion (positive $v s$. negative) & 0.882 & $0.354-2.292$ & 0.7884 & & & \\
\hline Lymph node metastasis (N2,3 vs. N0,1) & 0.889 & $0.370-2.111$ & 0.8889 & & & \\
\hline Liver metastasis (positive $v s$. negative) & 0.760 & $0.277-2.661$ & 0.6338 & & & \\
\hline Lung metastasis (positive $v s$. negative) & 0.954 & $0.359-2.310$ & 0.9191 & & & \\
\hline Peritoneal dissemination (positive $v s$. negative) & 1.257 & $0.512-2.979$ & 0.6073 & 1.049 & $0.358-3.250$ & 0.9299 \\
\hline miR-21 expression $(\geq 8.1$ vs. $<8.1)$ & 4.133 & $1.107-12.776$ & 0.0364 & 4.927 & $1.079-20.806$ & 0.0403 \\
\hline Number of organs affected by metastasis $(\geq 2 v s .1)$ & 0.863 & $0.353-2.160$ & 0.7458 & 0.748 & $0.233-2.432$ & 0.6210 \\
\hline
\end{tabular}

HR, Hazard ratio; CI, confidence interval; miR-21, microRNA-21.

Table V. Results of the uni/multivariate analysis for overall survival.

\begin{tabular}{|c|c|c|c|c|c|c|}
\hline & \multicolumn{3}{|c|}{ Univariate } & \multicolumn{3}{|c|}{ Multivariate } \\
\hline & OR & $95 \% \mathrm{CI}$ & $p$-Value & OR & $95 \% \mathrm{CI}$ & $p$-Value \\
\hline Age $(\geq 65 v s .<65)$ & 1.036 & $0.423-2.315$ & 0.9348 & & & \\
\hline Depth of tumor (T4 vs. T1-3) & 2.072 & $0.917-5.112$ & 0.0808 & 1.751 & $0.598-5.473$ & 0.3113 \\
\hline Histology (mucinous, poorly $v s$. well, moderately) & 2.232 & $0.507-7.026$ & 0.2536 & 1.634 & $0.305-7.373$ & 0.5408 \\
\hline Lymphatic vessel invasion (positive $v s$. negative) & 1.083 & $0.217-19.636$ & 0.9378 & & & \\
\hline Blood vessel invasion (positive vs. negative) & 1.198 & $0.540-2.722$ & 0.6560 & & & \\
\hline Lymph node metastasis (N2,3 vs. N0,1) & 1.902 & $0.837-4.494$ & 0.1247 & & & \\
\hline Liver metastasis (positive $v s$. negative) & 0.459 & $0.174-1.432$ & 0.1658 & & & \\
\hline Lung metastasis (positive $v s$. negative) & 1.542 & $0.653-3.408$ & 0.3098 & & & \\
\hline Peritoneal dissemination (positive $v s$. negative) & 1.419 & $0.612-3.151$ & 0.4028 & 0.795 & $0.273-2.260$ & 0.6659 \\
\hline miR-21 expression $(\geq 8.1 \mathrm{vs} .<8.1)$ & 2.670 & $0.765-7.238$ & 0.1131 & 2.522 & $0.649-8.341$ & 0.1681 \\
\hline Number of organs affected by metastasis ( $\geq 2 v s .1)$ & 1.305 & $0.593-3.016$ & 0.5118 & 1.029 & $0.346-3.151$ & 0.9589 \\
\hline
\end{tabular}

HR, Hazard ratio; CI, confidence interval; miR-21, microRNA-21.

PDCD4, which increases in apoptotic tumor tissue, is a well-known tumor suppressor protein (25-28). PDCD4 is a target protein of miR-21 and miR-21 has been shown to reduce PDCD4 expression (10). PDCD4 inhibits tumorigenesis (28), tumor progression (28), tumor invasion (29) and proliferation (30), as increasing miR-21 expression has been shown to result in tumorigenesis, tumor progression, tumor invasion and proliferation.

High miR-21 levels in the stroma of cancer correlate with tumor progression (22). In this study, we extracted microRNAs from tumor tissue, included in not only tumor cells but also the stroma. Consequently, we evaluated miR21 levels in both cancer cells and tumor tissue, including stroma, lymphocytes, fibroblasts, myofibroblasts, etc. MiR21 in fibroblasts and myofibroblasts inhibits the expression of reversion-inducing cysteine-rich protein with Kazal motifs
(RECK), which increases matrix metalloproteinase 2 (MMP2 ). Degradation of type IV collagen in the extracellular matrix by MMP-2 causes tears in the basal membrane, allowing cancer cells to obtain motility. Therefore, MMP-2 induces tumor invasion and metastasis (21). MiR-21 induced MMP-2 via inhibiting RECK expression, as miR-21 is concerned with tumor invasion and metastasis. As described above, miR-21, which is elevated in tumor tissues (including the stroma), is associated with OS.

Resistance to chemotherapy and cancer progression are factors associated with prognosis. In this study, miR-21 expression was associated with the resistance to chemotherapy and cancer progression. Although we could not clarify which mechanism most influenced clinical outcome, each factor might have an interdependent role in a worse prognosis with unresectable metastatic colon cancer. 
This study is associated with some limitations since it was retrospective in nature and included a relatively small number of patients. Also, we set 8.1 as the cut-off value of the relative expression level of miR-21 because the score was optimal for PFS. However, the appropriate cut-off value of miR-21 expression remains unclear. Further studies, including prospective studies with larger cohorts, should, thus, be performed to confirm our findings and determine the appropriate cut-off value of miR-21 expression.

\section{Conclusion}

This study demonstrated that miR-21 expression might be a useful prognostic factor for estimating the survival and chemotherapeutic outcomes in patients with unresectable metastatic colon cancer.

\section{Conflicts of Interest}

The Authors declare that they have no competing interests.

\section{Acknowledgements}

This research received no specific grants from any funding agency in the public, commercial or not-for-profit sectors. We thank Brian Quinn who provided medical writing services on behalf of JMC, Ltd.

\section{References}

1 Matsuda A, Matsuda T, Shibata A, Katanoda K, Sobue T and Nishimoto H: Cancer incidence and incidence rates in Japan in 2008: A study of 25 population-based cancer registries for the monitoring of cancer incidence in Japan (MCIJ) project. Jpn J Clin Oncol 44: 388-396, 2014.

2 Heinemann V, von Weikersthal LF, Decker T, Kiani A, VehlingKaiser U, Al-Batran SE, Heintges T, Lerchenmüller C, Kahl C, Seipelt G, Kullmann F, Stauch M, Scheithauer W, Hielscher J, Scholz M, Müller S, Link H, Niederle N, Rost A, Höffkes HG, Moehler M, Lindig RU, Modest DP, Rossius L, Kirchner T, Jung A and Stintzing S: FOLFIRI plus cetuximab versus FOLFIRI plus bevacizumab as first-line treatment for patients with metastatic colorectal cancer (FIRE-3): A randomised, open-label, phase 3 trial. Lancet Oncol 15: 1065-1075, 2014.

3 Loupakis F, Cremolini C, Masi G, Lonardi S, Zagonel V, Salvatore L, Cortesi E, Tomasello G, Ronzoni M, Spadi R, Zaniboni A, Tonini G, Buonadonna A, Amoroso D, Chiara S, Carlomagno C, Boni C, Allegrini G, Boni L and Falcone A: Initial therapy with FOLFOXIRI and bevacizumab for metastatic colorectal cancer. N Engl J Med 371: 1609-1618, 2014.

4 National Comprehensive Cancer Network: NCCN practice guidelines in oncology (NCCN guidelines) colon cancer, Version 2.2015. http://www.tri-kobe.org/nccn/guideline/colorectal/japanese/ colon.pdf (Accessed June 26, 2015).

5 Filipowicz W, Bhattacharyya SN and Sonenberg N: Mechanisms of post-transcriptional regulateon by microRNAs: Are the answer in sight? Nat Rev Genetics 9: 102-114, 2008.
6 Zhang B, Pan X, Cobb GP and Anderson TA: MicroRNAs as oncogenes and tumor suppressors. Deveropmental Biol 302: 1$12,2007$.

7 Valeri N, Gasparini P, Braconi C, Paone A, Lovat F, Fabbri M, Sumani KM, Alder H, Amadori D, Patel T, Nuovo GJ, Fishel R and Croce CM: MicroRNA-21 induces resistance to 5-fluoroutacil by down-regulating human DNA MutS homolog 2(hMSH2). Proc Natl Acad Sci USA 107(49): 21098-21103, 2010.

8 Liu K, Li G, Fan C, Zhou X, Wu B and Li J.: Increased expression of microRNA-21 and its association with chemotherapeutic response in human colorectal cancer. J Int Medical Res 39: 2288-2295, 2011.

9 Yu Y, Kanwar SS, Patel BB, Oh PS, Nautiyal J, Sarkar FH and Majumdar AP: MicroRNA-21 induces stemness by downregulating transforming growth factor betareceptor 2 (TGF $\beta R 2$ ) in colon cancer cells. Carcinogenesis 33(1): 68-76, 2012.

10 Deng J, Lei W, Fu JC, Zhang L, Li JH and Xiong JP: Targeting miR-21 enhances the sensitivity of human colon cancer HT-29 cells to chemoradiotherapy in vitro. Biochem and Biophys Res Commun 443(3): 789-795, 2014.

11 Asangani IA, Rasheed SA, Nikolova DA, Leupold JH, Colburn $\mathrm{NH}$, Post $\mathrm{S}$ and Allgayer H: MicroRNA-21(miR-21) posttranscriptionally down regulates tumor suppressor Pdcd4 and stimulates invasion, intravasation and metastasis in colorectal cancer. Oncogene 27: 2128-2136, 2008.

12 Therasse P, Arbuck SG, Eisenhauer EA, Wanders J, Kaplan RS, Rubinstein L, Verweij J, Van Glabbeke M, van Oosterom AT, Christian MC and Gwyther SG: New guidelines to evaluate the response to treatment in solid tumors (RECIST Guidelines). J Natl Cancer Inst 92: 205-216, 2000.

13 Fishel R: The selection for mismatch repair defects in hereditary nonpolyposis colorectal cancer: Revising the mutator hypothesis. Cancer Res 61: 7369-7374, 2001.

14 Meyers M: DNA mismatch repair-dependent response fluoropyrimidine-generated damage. J Biol Chem 280: 55165526, 2005.

15 Hewish M: Mismatch repair deficient colorectal cancer in the era of personalized treatment Nat Rev Clin Oncol 7(4): 197-208, 2010.

16 Maehama T and Dixon JE: The tumor suppressor, PTEN/ MMAC1, dephosphorylates the lipid second messenger, phosphatidylinositol 3,4,5-triphosphate. J Biol Chem 273: 13375-13378, 1998.

17 Di Cristofano A and Pandolfi PP: The multiple roles of PTEN in tumor suppression. Cell 100: 387-390, 2000.

18 Frattini M, Saletti P, Romagnani E, Martin V, Molinari F, Ghisletta M, Camponovo A, Etienne LL, Cavalli F and Mazzucchelli L: PTEN loss of expression predicts cetuximab efficacy in metastatic colorectal cancer patients. Br J Cancer 97: 1139-1145, 2007.

19 Yu Y, Kanwar SS, Patel BB, Nautiyal J, Sarkar FH and Majumdar PN: Elimination of colon cancer stem-like cells by the combination of curcuman and FOLFOX. Translational Oncol 2(4): 321-328, 2009.

20 Kanwar SS, Yu Y, Nautiyal J, Patel BB and Majumdar AP: The Wnt/beta-catenin pathway regulates growth and maintenance of colonospheres. Mol Cancer 9: 212, 2010.

$21 \mathrm{Li} \mathrm{XH}$ : Urothelial transdifferentiation to prostate epithelia is mediated by paracrine TGF-beta signaling. Differentiation 77 : 95-102, 2009. 
22 Bullock MD, Pickard KM, Nielsen BS, Sayan AE, Jenei V, Mellone M, Mitter R, Primrose JN, Thomas GJ, Packham GK and Mirnezami AH: Pleiotropic actions of miR-21 highlight the critical role of deregulated stromal microRNAs during colorectal cancer progression. Cell Death Dis 4: e684, 2013.

23 Kang WK, Lee JK, Oh ST, Lee SH and Jung CK: Stromal expression of miR-21 in T3-4a colorectal cancer is an independent predictor of early tumor relapse. BMC Gastroenterol 15: 2, 2015.

24 Meng F, Henson R, Wehbe-Janek H, Ghoshal K, Jacob ST and Patel T: MicroRNA-21 regulates expression of the PTEN tumor suppressor gene in human hepatocellular cancer. Gastroenterol 133: 647-658, 2007.

25 Cmarik JL, Min H, Hegamyer G, Zhan S, Kulesz-Martin M, Yoshinaga H, Matsuhashi S and Colburn NH: Differentially expressed protein Pdcd4 inhibits tumor promoter-induced neoplastic transformation. Proc Natl Acad Sci USA 96: 14037 14042, 1999.

26 Bitomsky N, Bohm M and Klempnauer KH: Transformation suppressor protein Pdcd4 interferes with JNK-mediated phosphorylation of c-Jun and recruitment of the coactivator p300 by c-Jun. Oncogene 23: 7484-7493, 2004.
27 Goke R, Barth P, Schmidt A, Samans B and Lankat-Buttgereit B: Programmed cell death protein 4 suppresses CDK1/cdc2 via induction of p21 (Waf1/Cip1) Am J Physiol Cell Physiol 287: 1541-1546, 2004.

28 Jansen AP, Camalier CE and Colburn NH: Epidermal expression of the translation inhibitor programmed cell death 4 suppresses tumorigenesis. Cancer Res 65: 6034-6041, 2005.

29 Leupold JH, Yang HS, Colburn NH, Asangani I, Post S and Allgayer $\mathrm{H}$ : Tumor suppressor Pdcd4 inhibits invasion/ intravasation and regulates urokinase receptor (u-PAR) gene expression via Sp-transcription factors. Oncogene 26: 45504562, 2007.

30 Chen Y, Knösel T, Kristiansen G, Pietas A, Garber ME, Matsuhashi S, Ozaki I and Petersen I: Loss of PDCD4 expression in human lung cancer correlates with tumour progression and prognosis. J Pathol 200: 640-646, 2003.

Received August 29, 2016

Revised September 16, 2016

Accepted September 19, 2016 